\title{
The next best time for demographers to contribute to climate change research
}

\author{
Anastasia J. Gage*
}

According to the medium variant projection of the United Nations World Population Prospects: 2015 Revision, the world population is on course to increase from 7.3 billion as of mid-2015 to 9.7 billion in 2050 and to 11.2 billion in 2100 (United Nations, 2015). Much of this growth will occur in developing countries, 35 of which are expected to experience a tripling of their populations between 2015 and 2100. These demographic trends and population dynamics have important, though complex, relationships with climate change itself, as well as with the vulnerability of populations to the negative effects of climate change, efforts to mitigate and adapt to climate change, development, and health.

To date, however, the contributions of demographers and population scientists to international discourse around the interrelationship between population and climate change have been rather limited. As the Population and Sustainability Network (2014) has noted, although the fields of population, development, global health, and climate change are highly interconnected, the collaboration between scholars from these fields has so far been insufficient. This failure to work together makes it difficult to consider how each of these areas of research is linked to climate change.

It is indisputable that population affects climate change. The results of an expert group meeting on population dynamics and climate change organized by the United Nations Population Fund (UNFPA) and the International Institute on Environment and Development (IIED) demonstrate clearly that the size, the structure, and the dynamics of populations influence and are influenced by climate change (UNFPA and IIED, 2009). So why are so few demographers participating actively in international policy discussions about potential responses to climate change? At a basic level, demographers seek to understand the size, composition, and flow of populations; as well as the biological, socioeconomic, political, and environmental processes that influence those changes. Many variables that can influence the

\footnotetext{
${ }^{*}$ Anastasia J. Gage, Department of Global Community Health and Behavioral Sciences, Tulane University School of Public Health and Tropical Medicine, New Orleans, LA 70112, USA Email: agage@tulane.edu
} 
trajectory of global greenhouse emissions-including urbanization, age structure, household size, and socioeconomic group - are squarely within the domain of demography. To date, these factors have been omitted from the Intergovernmental Panel on Climate Change Models of possible pathways for carbon dioxide emissions until the end of the 21st century; instead, these models have focused narrowly on population size and growth.

Understanding the links between population dynamics and climate change, and ensuring that conclusions regarding these relationships are evidence-based, will involve the identification, collection, and integration of data at the global, regional, and country levels. Demographers are well-positioned to provide substantive and methodological guidance and knowledge about population size and growth, emissions, population vulnerability and adaptation, migration, and urbanization; and to address the challenges inherent in incorporating economic, social, technological, and demographic data into climate change models.

Among the contributions demographers can make to such efforts are evaluating the quality of the relevant data, and promoting modelling for smaller geographic areas to facilitate local-level climate change adaptation and policy formulation. These contributions can help to meet the data analysis needs of policy-relevant research initiatives that seek answers to the following questions: Which populations will be affected? Which places are at risk? What are those risks? How do the risks vary among people and places? The analysis of the 2010 rounds of censuses will provide researchers with the opportunity to overlay climate-related risk maps onto population and socioeconomic data for small disaggregated administrative areas, which will allow them to make finer estimates of the numbers of people who are vulnerable to the impacts of climate change. Supporting the improvement of data streams is clearly within the scope of demography, as is addressing the challenges presented by indicator definitions, missing data, and the measurement of consumption-based versus supply-based emissions.

With more than half of the world's population currently living in urban areas, trends in urbanization have significant implications for consumption patterns, emissions, climate change, and urban adaptation in different parts of the world. Demographers have long explored the components of urban growth and changes in human settlement systems around the world. Yet a number of questions about how demographers are addressing these issues arise. For example, how are demographers contributing to our understanding of how the impact of climate change varies by type of settlement, location, population density, and growth? And, how are demographers shedding light on spatial variation in the vulnerability of the poor to the impacts of climate change, and on the roles of gender and migration?

The debates surrounding population and climate change are, of course, often heated. Although rapid population growth in the global south heightens vulnerability to climate change, the consumption levels and the generation of greenhouse gas emissions by industrialized countries-many of which are experiencing slow population growth or population stabilization-have historically been the key drivers of climate change. Is a desire to avoid being drawn into debates about 
the role of family planning programs and countries' rights to economic and social advancement leading demographers to downplay the effects of population trends, prospects, and dynamics on climate change, adaptation, and mitigation?

Demographers and population scientists are no strangers to complex and emotional policy controversies. At the 1974 World Population Conference in Bucharest there was sharp disagreement between representatives of developed countries who argued that rapid population growth impeded development, and representatives of developing countries who asserted that population problems are a consequence and not a cause of underdevelopment, and that the solution to these problems lies in the redistribution of resources in a new international economic order. In the current climate change debate, it is important for population scientists to bring to the table lessons learned since the 1994 International Conference on Population and Development about engaging governments to enable people to freely and responsibly choose the number and the spacing of their children by providing them with access to family planning information and contraceptive methods.

A fundamental question is whether and, if so, how the next generation of demographers is being prepared to meet the challenges associated with climate change. To what extent do individual demographers and demographic institutions have the capacity to conduct climate change and spatial analyses, and to integrate satellite imagery, climate modeling, demographic data, and socioeconomic data in studying conditions in the poorest countries of the world-i.e. in countries that are not among the major contributors of greenhouse gases currently, but that are likely to be hardest hit by the negative effects of climate change? Is it time for us to revisit the content of demography and population studies training programs?

I have always viewed the core work we do in demography as a means to an end: that is, providing the evidence needed to inform policies and programs and foster improvements in population well-being. However, recent developments have highlighted the need for demographers to become more active in addressing climate change and environmental degradation, as these phenomena threaten the well-being of current and future generations. There is, therefore, a window of opportunity for demographers to contribute to the ongoing discourse on climate and the environment.

It is time to start a dialogue on how demographers will contribute to assessments of the direct and indirect impact of climate change. It is time for demographers to help policy-makers, local leaders, and the public gain a better understanding of how climate change and environmental degradation are influenced by human populations, and how population health and well-being will in turn be influenced by climate change. There is an urgent need for demographic data to be used to support decision-makers in averting, adapting to, and possibly even reversing current warming trends. As demographers, will we help to shape future discourse, policies, and programmatic actions, or will we sit idly by and watch how the effects of climate change unfold?

The best time to plant a tree was 20 years ago. The next best time is now (Chinese proverb). 


\section{References}

Population and Sustainability Network 2014. 2014 Annual Report. Population and Sustainability Network. London: Population and Sustainability Network.

United Nations, Department of Economic and Social Affairs, Population Division (2015). World Population Prospects: The 2015 Revision. Key Findings and Advance Tables. Working Paper No. ESA/P/WP.241.

UNFPA and IIED 2009. Population Dynamics and Climate Change. New York: United Nations Population Fund. 